Research Article

\title{
Symplectic Approach of Three-Dimensional Palatini Theory Plus a Chern-Simons Term
}

\author{
Alberto Escalante (iD and Prihel Cavildo-Sánchez \\ Instituto de Física Luis Rivera Terrazas, Benemérita Universidad Autónoma de Puebla, Apartado Postal J-48, 72570 Puebla, \\ PUE, Mexico \\ Correspondence should be addressed to Alberto Escalante; aescalan@ifuap.buap.mx
}

Received 20 December 2017; Revised 13 February 2018; Accepted 26 February 2018; Published 29 March 2018

Academic Editor: Dimitrios Tsimpis

Copyright (C) 2018 Alberto Escalante and Prihel Cavildo-Sánchez. This is an open access article distributed under the Creative Commons Attribution License, which permits unrestricted use, distribution, and reproduction in any medium, provided the original work is properly cited.

\begin{abstract}
Using the symplectic framework of Faddeev-Jackiw, the three-dimensional Palatini theory plus a Chern-Simons term [P-CS] is analyzed. We report the complete set of Faddeev-Jackiw constraints and we identify the corresponding generalized Faddeev-Jackiw brackets. With these results, we show that although P-CS produces Einstein's equations, the generalized brackets depend on a Barbero-Immirzi-like parameter. In addition, we compare our results with those found in the canonical analysis showing that both formalisms lead to the same results.
\end{abstract}

\section{Introduction}

It is well known that the core of canonical gravity is based on the Hamiltonian formalism for singular systems developed by Dirac $[1,2]$. In fact, the Dirac formalism is a powerful approach for studying gauge systems and relevant information is obtained from it. For instance, it is possible to obtain the gauge symmetry, the counting of the physical degrees of freedom, and the identification of the quantum brackets, the so-called Dirac brackets. The modern starting point for studying the canonical approach of gravity is based on the Holst action, which depends on a connexion valued on the $\mathrm{SO}(3,1)$ group, a tetrad 1-form, and a parameter called the Barbero-Immirzi (the so-called $\gamma$ parameter) [3-5]. In fact, because of the presence of the $\gamma$ parameter, the Holst action provides a set of actions classically equivalent to Einstein's theory and its contribution can be appreciated at the classical level in the structure of the constraints. On the other hand, in the coupling of fermionic matter with gravity, $\gamma$ determines the coupling constant of a four-fermion interaction [5]. It is important to comment that Holst's action also allows us to reproduce the different classical formulations of canonical gravity reported in the literature; for instance, if $\gamma$ is real, then the Barbero formulation is obtained [6]; moreover, if the parameter is complex, then the Ashtekar approach is reproduced [7]. However, at the moment, the $\gamma$ parameter is controversial because its value is unknown. In fact, in the quantum version of the Barbero formulation, there is a contribution of $\gamma$ in the area operator $[8,9]$. Thus, if we were able to develop an experiment for calculating quanta of area, then we could calculate the value of $\gamma$.

On the other hand, in the three-dimensional case, there is a model with the same characteristics of the Holst action, the so-called Bonzom-Livine action [BL] [10]. In fact, the BL theory turns out to be classically equivalent to threedimensional Palatini theory. It provides a set of actions sharing the classical equations of motion with the Palatini theory, and its symplectic structure depends on a $\gamma$-like parameter. In this respect, it is also possible to obtain similar results from an action principle with a more simple structure than BL theory: the Palatini theory plus $1 / \gamma$ times the Chern-Simons term [P-CS]. In fact, the P-CS theory also provides a classical set of actions sharing the equations of motion with Einstein's theory and their symplectic structure depends on the $\gamma$ parameter. It is important to comment that the $[\mathrm{P}-\mathrm{CS}]$ has been analyzed from the Lagrangian and 
Hamiltonian points of view [10]; however, all the fundamental steps were ignored; that is, the complete structure of the constraints and the symmetries of the theory were not reported.

In this manner, with the antecedents mentioned above, in this paper, we will study the P-CS theory from a symplectic point of view. For this aim, we will use the symplectic formalism of Faddeev-Jackiw [FJ] [11-25], due basically to the fact that the FJ approach is more economical than Dirac's method. In fact, the FJ is a symplectic description where all relevant information of the theory can be obtained through a symplectic tensor, which is constructed from the symplectic variables that are identified from the Lagrangian. If the theory is singular, then there will be constraints and the FJ method avoids the classification of the constraints in primary, secondary, first class, or second class, as it is done in Dirac's method. Furthermore, from the components of the symplectic tensor, it is possible to identify the FJ generalized brackets; at the end of the calculations, the Dirac and the FJ generalized brackets are equivalent. Furthermore, in order to complete our analysis, we have added as an appendix the Dirac approach of P-CS, where we report the complete structure of the constraints and at the end we compare the results obtained in both formulations.

The paper is organized as follows. In Section 2, the symplectic analysis is developed; we report the complete FJ constraints and a symplectic tensor is constructed. From that symplectic tensor, the generalized FJ brackets are identified and we compare the FJ results with those obtained in the Dirac method. In Section 3, we present prospects and conclusions. Finally, an appendix is added resuming the canonical analysis.

\section{Symplectic Analysis}

The theory that we will analyze along this paper is described by the following action:

$$
\begin{aligned}
S[e, A]= & 2 \int_{M} e^{i} \wedge F_{i}[A] \\
& +\frac{1}{\gamma} \int_{M}\left[A^{i} \wedge d A_{i}+\frac{2}{3} \epsilon_{i j k} A^{i} \wedge A^{j} \wedge A^{k}\right],
\end{aligned}
$$

where $M$ is a three-dimensional manifold without boundary, $F=d A+A \wedge A$ is the curvature of the connexion $A, e$ 's are the triad fields, $i, j, k, \ldots=0,1,2$ are internal $\mathrm{SU}(2)$ indices, and $\gamma$ is an arbitrary parameter like a Barbero-Immirzi parameter [10]. From the variation of the action, the following equations of motion arise:

$$
D e+\frac{1}{\gamma} F=0, \quad F=0
$$

which are classically equivalent to the three-dimensional Einstein equations. Along the paper, we will use the notation $\mu, \nu, \ldots=0,1,2$ for space-time indices and the alphabet letters $a, b, c$ for space indices. Moreover, we will suppose that the manifold has topology $M=\Sigma \times R$, where $\Sigma$ is a Cauchy surface and $R$ is an evolution parameter. With these considerations at hand, we perform the $2+1$ decomposition, and action (1) takes the following form:

$$
\begin{aligned}
& S[e, A]=\int \epsilon^{0 a b}\left[\left(e_{b}^{i}+\frac{1}{2 \gamma} A_{b}^{i}\right) \dot{A}_{a i}+\frac{1}{2} e_{0}^{i} F_{a b i}\right. \\
& \left.+A_{0 i}\left(D_{a} e_{b}^{i}+\frac{1}{2 \gamma} F_{a b}{ }^{i}\right)\right] d^{3} x,
\end{aligned}
$$

where

$$
\begin{aligned}
F_{a b i} & =\partial_{a} A_{b i}-\partial_{b} A_{a i}+\epsilon_{i}^{j k} A_{a j} A_{b k}, \\
D_{a} e_{b i} & =\partial_{a} e_{b i}+\epsilon_{i}{ }^{j k} A_{a j} e_{b k} .
\end{aligned}
$$

From (3), we can identify that the Lagrangian density has the following symplectic form [11-25]:

$$
\begin{aligned}
\mathscr{L}^{(0)} & \\
= & \epsilon^{0 a b}\left(e_{b}{ }^{i}+\frac{1}{2 \gamma} A_{b}{ }^{i}\right) \dot{A}_{a i}+\frac{1}{2} \epsilon^{0 a b} e_{0}{ }^{i} F_{a b i} \\
& +\epsilon^{0 a b} A_{0 i}\left(D_{a} e_{b}{ }^{i}+\frac{1}{2 \gamma} F_{a b}{ }^{i}\right) \\
= & \epsilon^{0 a b}\left(e_{b}{ }^{i}+\frac{1}{2 \gamma} A_{b}{ }^{i}\right) \dot{A}_{a i} \\
& -\left[-\frac{1}{2} \epsilon^{0 a b} e_{0}{ }^{i} F_{a b i}-\epsilon^{0 a b} A_{0 i}\left(D_{a} e_{b}{ }^{i}+\frac{1}{2 \gamma} F_{a b}{ }^{i}\right)\right] \\
= & a_{i}^{(0)} \dot{\xi}^{(0) i}-\mathscr{V}^{(0)},
\end{aligned}
$$

where $\xi^{(0) i}$ are the set of symplectic variables,

$$
\xi^{(0) i}=\left(e_{0 i}, e_{a i}, A_{0 i}, A_{a i}\right),
$$

$a^{(0)}$ 's are the corresponding 1-forms

$$
a_{i}^{(0)}=\left(0,0,0, \epsilon^{0 a b}\left(e_{b}^{i}+\frac{1}{2 \gamma} A_{b}^{i}\right)\right),
$$

and in addition $\mathscr{V}^{(0)}$ is the so-called symplectic potential:

$$
\mathscr{V}^{(0)}=-\frac{1}{2} \epsilon^{0 a b} e_{0}{ }^{i} F_{a b i}-\epsilon^{0 a b} A_{0 i}\left(D_{a} e_{b}{ }^{i}+\frac{1}{2 \gamma} F_{a b}{ }^{i}\right) .
$$

In the FJ context, the equations of motion are expressed as $f_{i j}^{(0)} \dot{\xi}^{(0) j}=\partial \mathscr{V}^{(0)} / \partial \xi^{(0) i}$, where $f_{i j}^{(0)}$ is the so-called symplectic matrix and it is given by $f_{i j}^{(0)}=\partial a_{j}^{(0)} / \partial \xi^{(0) i}-\partial a_{i}^{(0)} / \partial \xi^{(0) j}[11-$ 25]. In this manner, using the symplectic variables and the 1 -forms given above, the symplectic matrix acquires the form 


$$
f_{i j}^{(0)}=\left(\begin{array}{cccc}
0 & 0 & 0 & 0 \\
0 & 0 & 0 & \epsilon^{0 a b} \delta^{i l} \\
0 & 0 & 0 & 0 \\
0 & -\epsilon^{0 a b} \delta^{i l} & 0 & \frac{1}{\gamma} \epsilon^{0 a b} \delta^{i l}
\end{array}\right) \delta^{2}(x-y) .
$$

Furthermore, we observe that $f_{i j}^{(0)}$ is a singular matrix; hence, this means that the Lagrangian describes a constrained theory; therefore, there will be constraints. In order to identify these FJ constraints, we calculate the zero modes of $f_{i j}^{(0)}$ :

$$
\begin{aligned}
& V_{1}^{(0)}=\left(V^{e_{0 i}}, 0,0,0\right), \\
& V_{2}^{(0)}=\left(0,0, V^{A_{0 i}}, 0\right),
\end{aligned}
$$

where $V^{e_{0 i}}$ and $V^{A_{0 i}}$ are arbitrary functions. In this manner, the FJ constraints are found from the following contractions [11-25]:

$$
\begin{aligned}
\Omega_{1}^{(0)} & =\int \mathbf{d}^{3} x V_{1}^{(0)^{i}} \frac{\delta}{\delta \xi^{(0)^{i}}} \int \mathbf{d}^{3} y \mathscr{V}^{(0)}(\xi) \\
& =\int \mathbf{d}^{3} x V^{e_{0 i}} \int \frac{\delta \mathscr{V}^{(0)}(x)}{\delta e_{0 i}(y)} d^{2} y \\
& =V^{e_{0 i}}\left[-\frac{1}{2} \epsilon^{0 a b} F_{a b}^{i}\right]=0 . \\
\Omega_{2}^{(0)} & =\int \mathbf{d}^{3} x V_{2}^{(0)^{i}} \frac{\delta}{\delta \xi^{(0)^{i}}} \int \mathbf{d}^{3} y \mathscr{V}^{(0)}(\xi)
\end{aligned}
$$

$$
\begin{aligned}
& =\int \mathbf{d}^{3} x V^{A_{0 i}} \int \frac{\delta \mathscr{V}^{(0)}(x)}{\delta A_{0 i}(y)} d^{2} y \\
& =-V^{A_{0 i}} \epsilon^{0 a b}\left[D_{a} e_{b}{ }^{i}+\frac{1}{2 \gamma} F_{a b}{ }^{i}\right]=0,
\end{aligned}
$$

In this manner, the FJ constraints read

$$
\begin{aligned}
& \Omega_{1}^{(0) i}=\frac{1}{2} \epsilon^{0 a b} F_{a b}{ }^{i}=0, \\
& \Omega_{2}^{(0) i}=\epsilon^{0 a b}\left[D_{a} e_{b}{ }^{i}+\frac{1}{2 \gamma} F_{a b}{ }^{i}\right]=0 .
\end{aligned}
$$

Now, we will determine whether there are more FJ constraints; for this aim, we require consistency of the constraints [11-25]. This means that the following system is satisfied:

$$
\bar{f}_{i j}^{(0)} \dot{\xi}^{j}=Z_{i}
$$

where

$$
\begin{aligned}
\bar{f}_{i j}^{(0)} & =\left(\begin{array}{c}
f_{i j}^{(0)} \\
\frac{\partial \Omega^{(0)}}{\partial \xi^{j}}
\end{array}\right), \\
Z_{i} & =\left(\begin{array}{c}
\frac{\partial \mathscr{V}^{(0)}}{\partial \xi^{i}} \\
0
\end{array}\right) .
\end{aligned}
$$

In this manner, the matrix $\bar{f}_{i j}^{(0)}$ has the explicit form

$$
\bar{f}_{i j}^{(0)}=\left(\begin{array}{cccc}
0 & 0 & 0 & 0 \\
0 & 0 & 0 & \epsilon^{0 a b} \delta^{i j} \\
0 & 0 & 0 & 0 \\
0 & -\epsilon^{0 a b} \delta^{i j} & 0 & \frac{1}{\gamma} \epsilon^{0 a b} \delta^{i j} \\
0 & 0 & 0 & \epsilon^{0 a b}\left[\delta^{i j} \partial_{b}+\epsilon^{i j k} A_{b k}\right] \\
0 \epsilon^{0 a b}\left[\delta^{i j} \partial_{b}+\epsilon^{i j k} A_{b k}\right] & 0 & \epsilon^{0 a b} \epsilon^{i j k} e_{b k}+\frac{1}{\gamma} \epsilon^{0 a b}\left[\delta^{i j} \partial_{b}+\epsilon^{i j k} A_{b k}\right]
\end{array}\right) \delta^{2}(x-y) .
$$

We can observe that this matrix is not square as expected; however, it has null vectors:

$$
\begin{aligned}
& v_{1}^{i}=\left(0, \partial_{i} V_{a}-\epsilon_{i}{ }^{j k} A_{a j} V_{k}, 0,0, V_{i}, 0\right), \\
& v_{2}^{i}=\left(0,-\epsilon_{i}{ }^{j k} e_{a j} V_{k}, 0, \partial_{a} V_{i}-\epsilon_{i}{ }^{j k} A_{a j} V_{k}, 0, V_{i}\right) .
\end{aligned}
$$

$$
Z_{i}=\left(\begin{array}{c}
\Omega_{1}^{(0) i} \\
-\epsilon^{0 a b}\left[\partial_{b} A_{0}^{i}+\epsilon^{i j k} A_{b j} A_{0 k}\right] \\
\Omega_{2}^{(0) i} \\
\epsilon^{0 a b} \partial_{a}\left(e_{0}^{i}+\frac{1}{\gamma} A_{0}^{i}\right)-\epsilon^{0 a b} \epsilon^{i j k} A_{0 j} e_{a k} \\
-\epsilon^{0 a b} \epsilon^{i j k}\left(e_{0 j}+\frac{1}{\gamma} A_{0 j}\right) A_{a k} \\
0 \\
0
\end{array}\right)
$$


In this manner, the contraction of the null vectors (16) with $Z_{i}$ yields identities. For example, from the contraction of the vector $v_{1}^{i}$ with $Z_{i}$, one obtains

$$
v_{1}^{i} Z_{i}=\epsilon^{i j k} V_{i} A_{0 j} \Omega_{1 k}^{(0)}=0,
$$

which implies that there are no more FJ constraints. As we have mentioned previously, in the FJ approach, it is not necessary to perform the classification of the constraints in first class, second class, and so forth, but all constraints are at the same level. Hence, the counting of degrees of freedom is carried out in the following form: there are 6 dynamical variables $A_{a}^{i}$ and 6 independent FJ constraints $\Omega_{1}^{(0) i}, \Omega_{2}^{(0) i}$; in this manner, the theory is devoid of degrees of freedom, and the theory is topological as expected.

Now, we will add to the symplectic Lagrangian the new constraints found above and we define a new symplectic Lagrangian, say $\mathscr{L}^{(1)}$ :

$$
\begin{aligned}
\mathscr{L}^{(1)} & =a_{i}^{(0)} \dot{\xi}^{(0) i}-\left.\mathscr{V}^{(0)}\right|_{\Omega_{1}=\Omega_{2}=0}-\Omega_{1 i}^{(0)} \dot{\alpha}^{i}-\Omega_{2 i}^{(0)} \dot{\beta}^{i} \\
& =\epsilon^{0 a b}\left(e_{b}{ }^{i}+\frac{1}{2 \gamma} A_{b}{ }^{i}\right) \dot{A}_{a i}-\Omega_{1 i}^{(0)} \dot{\alpha}^{i}-\Omega_{2 i}^{(0)} \dot{\beta}^{i}
\end{aligned}
$$

where we have added the FJ constraints via Lagrange multipliers; namely, $e_{0 i} \equiv \dot{\alpha}_{i}$ and $A_{0 i} \equiv \dot{\beta}_{i}$. Note that the symplectic potential vanishes $\left.\mathscr{V}^{(0)}\right|_{\Omega_{1}=\Omega_{2}=0}=0$; this result is expected because of the general covariance of the theory; all the dynamics of the system are governed by a symmetry. From the symplectic Lagrangian (19), we identify the set of new symplectic variables

$$
\xi^{(1) i}=\left(\alpha_{i}, e_{a i}, \beta_{i}, A_{a i}\right)
$$

and the following 1-forms:

$$
a_{i}^{(1)}=\left(-\Omega_{1}^{(0) i}, 0,-\Omega_{2}^{(0) i}, \epsilon^{0 a b}\left(e_{b}^{i}+\frac{1}{2 \gamma} A_{b}^{i}\right)\right) \text {. }
$$

With the help of these symplectic variables, we calculate the new symplectic matrix; namely, $f_{i j}^{(1)}=\partial a_{j}^{(1)} / \partial \xi^{(1) i}-$ $\partial a_{i}^{(1)} / \partial \xi^{(1) j}$, which acquires the following explicit form:

$$
f_{i j}^{(1)}=\left(\begin{array}{cccc}
0 & 0 & 0 & {\left[\delta^{i j} \partial_{a}+\epsilon^{i j k} A_{a k}\right]} \\
0 & 0 & {\left[\delta^{i j} \partial_{a}-\epsilon^{i j k} A_{a k}\right]} & \delta^{i j} \\
0 & {\left[\delta^{i j} \partial_{a}+\epsilon^{i j k} A_{a k}\right]} & 0 & \epsilon^{i j k} e_{a k}+\frac{1}{\gamma}\left[\delta^{i j} \partial_{a}+\epsilon^{i j k} A_{a k}\right] \\
{\left[\delta^{i j} \partial_{a}-\epsilon^{i j k} A_{a k}\right]} & \delta^{i j} & -\epsilon^{i j k} e_{a k}+\frac{1}{\gamma}\left[\delta^{i j} \partial_{a}-\epsilon^{i j k} A_{a k}\right] & \frac{1}{\gamma} \delta^{i j}
\end{array}\right)
$$

This matrix is singular, and the corresponding zero modes are the generators of the gauge transformations. The null vectors are given by $\widetilde{v}^{(1)}=\left(0,-\epsilon_{l}{ }^{j k} \omega_{j} e_{a k}, \omega_{l}, \partial_{a} \omega_{l}-\epsilon_{l}{ }^{j k} \omega_{j} A_{a k}\right)$ and $\widetilde{v}^{(2)}=\left(-\tau_{i}, \partial_{a} \tau_{i}-\epsilon_{i}{ }^{j k} \tau_{j} A_{a k}, 0,0\right)$, where $\omega_{i}$ and $\tau_{i}$ form a set of infinitesimal parameters characterizing the gauge transformations. In fact, the null vectors generate the following gauge transformations:

$$
\begin{aligned}
\delta \alpha_{i} & =-\dot{\tau}_{i}, \\
\delta e_{a i} & =D_{a} \tau_{i}+\epsilon_{i}^{j k} e_{a j} \omega_{k}, \\
\delta \beta_{i} & =\dot{\omega} \\
\delta A_{a}^{i} & =D_{a} \omega^{i},
\end{aligned}
$$

where we can see that $\widetilde{v}^{(1)}$ is the generator of rotations and $\widetilde{v}^{(2)}$ of translations. Furthermore, the matrix (22) is still singular; however, we have shown that there are no more constraints and that the theory has a gauge symmetry. In order to construct a symplectic tensor, we need to fix the gauge [11-25], and thus we will fix the temporal gauge, say, $e_{0 i}=A_{0 i}=0$, which means that $\dot{\alpha}_{i}=0$ and $\dot{\beta}_{i}=0$. In this manner, the fixing gauge will be added to the symplectic Lagrangian via Lagrange multipliers, $\Theta^{i}$ and $\Xi^{i}$. Hence, the symplectic Lagrangian which will be called $\mathscr{L}^{(2)}$ is

$$
\begin{aligned}
\mathscr{L}^{(2)}= & a_{i}^{(2)} \dot{\xi}^{(2) i} \\
= & \epsilon^{0 a b}\left(e_{b}{ }^{i}+\frac{1}{2 \gamma} A_{b}{ }^{i}\right) \dot{A}_{a i}-\left(\Omega_{1}^{(0) i}+\Theta^{i}\right) \dot{\alpha}_{i} \\
& -\left(\Omega_{2}^{(0) i}+\Xi^{i}\right) \dot{\beta}_{i},
\end{aligned}
$$

and from this expression the following symplectic variables and the 1-forms are identified:

$$
\begin{aligned}
\xi^{(2) i} & =\left(\alpha_{i}, e_{a i}, \beta_{i}, A_{a i}, \Theta_{i}, \Xi_{i}\right) \\
a^{(2) i} & =\left(-\left(\Omega_{1}^{(0) i}+\Theta^{i}\right), 0\right. \\
& \left.-\left(\Omega_{2}^{(0) i}+\Xi^{i}\right), \epsilon^{0 a b}\left(e_{b}^{i}+\frac{1}{2 \gamma} A_{b}^{i}\right), 0,0\right) .
\end{aligned}
$$

In this manner, using these sets of symplectic variables and 1-forms, the corresponding symplectic matrix $f_{i j}^{(2)}$ is given by 


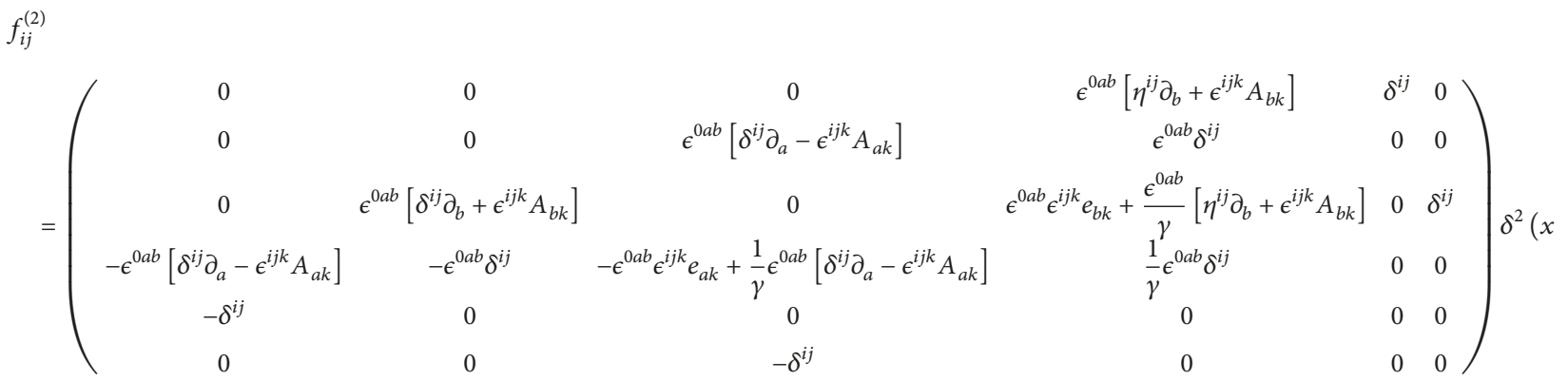

$$
\begin{aligned}
& -y) \text {, }
\end{aligned}
$$

where we note that $f_{i j}^{(2)}$ is not singular; therefore, there exists its inverse. After long calculations, the inverse of $f_{i j}^{(2)}$ is given by

$$
\left(f^{(2)}\right)_{i j}^{-1}=\left(\begin{array}{cccccc}
0 & 0 & 0 & 0 & -\delta_{i j} & 0 \\
0 & -\frac{1}{\gamma} \epsilon_{0 a b} \delta_{i j} & 0 & -\epsilon_{0 a b} \delta_{i j} & {\left[\delta_{i j} \partial_{b}+\epsilon_{j i}{ }^{k} A_{b k}\right]} & -\epsilon_{j i}{ }^{k} e_{a k} \\
0 & 0 & 0 & 0 & 0 & -\delta_{i j} \\
0 & \epsilon_{0 a b} \delta_{i j} & 0 & 0 & 0 & -\delta_{i j} \partial_{a}-\epsilon_{j i}{ }^{k} A_{a k} \\
\delta_{i j}-\left[\delta_{i j} \partial_{b}+\epsilon_{j i}{ }^{k} A_{b k}\right] & 0 & 0 & 0 & \epsilon^{0 a b} A_{a i} A_{b j} \\
0 & \epsilon_{j i}{ }^{k} e_{b k} & \delta_{i j} \delta_{i j} \partial_{a}+\epsilon_{j i}{ }^{k} A_{a k} & -\epsilon^{0 a b} A_{a i} A_{b j} & \epsilon^{0 a b} A_{a i} A_{b j}
\end{array}\right) \times \delta^{2}(x-y) .
$$

This corresponds to a symplectic tensor. From the symplectic tensor (27), we can identify the generalized FJ brackets by means of

$$
\left\{\xi_{i}^{(2)}(x), \xi_{j}^{(2)}(y)\right\}_{\mathrm{FJ}}=\left[f_{i j}^{(2)}(x, y)\right]^{-1}
$$

and hence the FJ brackets are given by

$$
\begin{gathered}
\left\{e_{a i}(x), e_{b j}(y)\right\}_{\mathrm{FJ}}=-\frac{1}{\gamma} \epsilon_{0 a b} \delta_{i j} \delta^{2}(x-y), \\
\left\{e_{a i}(x), A_{b j}(y)\right\}_{\mathrm{FJ}}=-\epsilon_{0 a b} \delta_{i j} \delta^{2}(x-y), \\
\left\{A_{a i}(x), A_{b j}(y)\right\}_{\mathrm{FJ}}=0,
\end{gathered}
$$

and we can observe that these brackets are the same as those obtained using the Dirac method (see the Appendix and [10]). Furthermore, we can observe that there is a $\gamma$-contribution in the fundamental brackets, which makes a difference with respect to Palatini theory [26]. In fact, in Palatini theory, the Dirac brackets between the triad fields are commutative, while in P-CS, they are not; however, we observe that P-CS and Palatini are equivalent in the limit when $\gamma$ goes to infinity.

\section{Conclusions}

In this paper, a symplectic analysis of P-CS has been performed. We reported the complete set of FJ constraints, the gauge symmetry, and the FJ generalized brackets which are not reported in the literature. The advantage for applying the symplectic formalism becomes to be present in a more economical analysis. In fact, it is not necessary to develop the classification of the constraints as in Dirac's approach (see the Appendix), and the results found in the Dirac method are reproduced in the symplectic scheme. Furthermore, we observed that the generalized brackets depend on $\gamma$, and this fact makes the P-CS theory different from Palatini theory. In fact, it is well known that two theories sharing the same equations of motion do not imply that these theories are equivalent at all [27-29]; the difference between the generalized brackets will be relevant in the quantization program; we need to remember that the symplectic structure is an essential ingredient in the quantum canonical approach [30]. It is important to note that the contribution of degrees of freedom just like the addition of matter fields could help us understand the nature of the $\gamma$ parameter. In fact, it is reported in the literature that, in the description of gravity coupled to fermion fields, the Immirzi parameter is a coupling constant determining the strength of a four-fermion interaction [31]. Hence, we expect that in three-dimensional coupling of $\mathrm{P}$ CS with matter the symplectic structure will not change and 
therefore we could investigate the relation between $\gamma$ and matter coupling through their symplectic structure and the constraints. All these ideas are already under study and will be the subject of forthcoming works.

\section{Appendix}

\section{Hamiltonian Analysis}

In this section, we report the principal results of the Hamiltonian analysis. We start with the following action:

$$
\begin{aligned}
& S[e, A]=\int \epsilon^{0 a b}\left[\left(e_{b}{ }^{i}+\frac{1}{2 \gamma} A_{b}{ }^{i}\right) \dot{A}_{a i}+\frac{1}{2} e_{0}{ }^{i} F_{a b i}\right. \\
& \left.+A_{0 i}\left(D_{a} e_{b}^{i}+\frac{1}{2 \gamma} F_{a b}{ }^{i}\right)\right] d^{3} x,
\end{aligned}
$$

where $\epsilon^{0 a b} \equiv \eta^{a b}$.

By introducing the canonical momenta to the complete set of dynamical variables $(e, A)$, given by

$$
\begin{gathered}
p^{\alpha}{ }_{i}=\frac{\partial \mathscr{L}}{\partial \dot{e}_{\alpha}{ }^{i}}, \\
\pi^{\alpha}{ }_{i}=\frac{\partial \mathscr{L}}{\partial \dot{A}_{\alpha}{ }^{i}},
\end{gathered}
$$

the following results are found: the theory presents 12 firstclass constraints

$$
\begin{aligned}
& \gamma_{1 i}^{0} \equiv p^{0}{ }_{i} \approx 0, \\
& \gamma_{2 i}^{0} \equiv \pi^{0}{ }_{i} \approx 0, \\
& \gamma_{1 i} \equiv \frac{1}{2} \eta^{a b} F_{a b i}+D_{a} p^{a}{ }_{i} \approx 0, \\
& \gamma_{2 i} \equiv D_{a} \pi^{a}{ }_{i}+\frac{1}{2 \gamma} \epsilon^{0 a b} \partial_{a} A_{b i}+\epsilon_{i j k} e_{a}{ }^{j} p^{a k} \approx 0
\end{aligned}
$$

and the following 12 second-class constraints:

$$
\begin{aligned}
& \chi_{1 i}^{a} \equiv p_{i}^{a} \approx 0, \\
& \chi_{2 i}^{a} \equiv \pi_{i}^{a}-\epsilon^{0 a b}\left(e_{b i}+\frac{1}{2 \gamma} A_{b i}\right) \approx 0 .
\end{aligned}
$$

The complete structure of these constraints is not reported in the literature. The algebra between the constraints is as follows:

$$
\begin{aligned}
& \left\{\gamma_{1 i}(x), \gamma_{1 j}(y)\right\}=0, \\
& \left\{\gamma_{1 i}(x), \gamma_{2 j}(y)\right\}=\epsilon_{i j}{ }^{k} \gamma_{1 k} \approx 0, \\
& \left\{\gamma_{1 i}(x), \chi_{1}^{a j}(y)\right\}=0, \\
& \left\{\gamma_{1 i}(x), \chi_{2}^{a j}(y)\right\}=\epsilon_{i}^{j k} \chi_{1 k}^{a} \approx 0, \\
& \left\{\gamma_{2 i}(x), \gamma_{2 j}(y)\right\}=\epsilon_{i l}{ }^{k} \gamma_{2 k} \approx 0, \\
& \left\{\gamma_{2 i}(x), \chi_{1}^{a j}(y)\right\}=\epsilon_{i}^{j k} \chi_{1 k}^{a},
\end{aligned}
$$

$$
\begin{aligned}
& \left\{\gamma_{2 i}(x), \chi_{2}^{a j}(y)\right\}=-\epsilon_{i}^{k j} \chi_{2 k}^{a} \approx 0, \\
& \left\{\chi_{1}^{a i}(x), \chi_{1}^{b j}(y)\right\}=0, \\
& \left\{\chi_{1}^{a i}(x), \chi_{2}^{b j}(y)\right\}=-\epsilon^{0 a b} \delta^{i j}, \\
& \left\{\chi_{2}^{a i}(x), \chi_{2}^{b j}(y)\right\}=-\frac{1}{\gamma} \epsilon^{0 a b} \delta^{i j},
\end{aligned}
$$

where we can see from the algebra that the constraint $\gamma_{1 i}$ is the generator of translations and $\gamma_{2 i}$ is the generator of rotations. For the counting of degrees of freedom, we observe that there are 36 canonical variables, 12 independent firstclass constraints, and 12 second-class constraints, and thus the theory lacks physical degrees of freedom.

The presence of second-class constraints implies the introduction of Dirac's brackets. The Dirac brackets between two functionals, say $A, B$, are defined by

$$
\begin{aligned}
\{A(x), B(y)\}_{D}=\{ & A(x), B(y)\}-\int d u d v \\
& \cdot\left\{A(x), \chi^{\alpha}(u)\right\} C_{\alpha \beta}^{-1}(u, v) \\
& \cdot\left\{\chi^{\beta}(v), B(y)\right\},
\end{aligned}
$$

where $\{A(x), B(y)\}$ is the Poisson bracket between the functionals $A, B$; the matrix $C_{\alpha \beta} \equiv\left\{\phi^{\alpha}, \phi^{\beta}\right\}$; here, $\left\{\phi^{\alpha}\right\}$ is the complete set of second-class constraints found above; and $C^{-1}$ represents the inverse of $C$. Using the second-class constraints, the following Dirac brackets are found:

$$
\begin{aligned}
& \left\{e^{a}{ }_{i}(x), e^{b}{ }_{j}(x)\right\}_{D}=-\frac{1}{\gamma} \delta_{i j} \epsilon^{0 a b} \delta^{2}(x-y), \\
& \left\{p^{a}{ }_{i}(x), p^{b}{ }_{j}(y)\right\}_{D}=0, \\
& \left\{e^{a}{ }_{i}(x), p^{b}{ }_{j}(y)\right\}_{D}=0, \\
& \left\{e^{a}{ }_{i}(x), A_{j}^{b}(y)\right\}_{D}=-\delta_{i j} \epsilon^{0 a b} \delta^{2}(x-y), \\
& \left\{A^{a}{ }_{i}(x), A^{b}{ }_{j}(y)\right\}_{D}=0, \\
& \left\{\pi^{a}{ }_{i}(x), \pi^{b}{ }_{j}(y)\right\}_{D}=0, \\
& \left\{A^{a}{ }_{i}(x), \pi^{b}{ }_{j}(y)\right\}_{D}=\delta_{i j} \epsilon^{0 a b} \delta^{2}(x-y), \\
& \left\{e^{a}{ }_{i}(x), \pi^{b}{ }_{j}(y)\right\}_{D}=-\frac{1}{2 \gamma} \delta_{i j} \epsilon^{0 a b} \delta^{2}(x-y), \\
& \left\{A_{i}^{a}(x), p^{b}{ }_{j}(y)\right\}_{D}=0,
\end{aligned}
$$

where we can observe that these brackets and the FJ coincide with each other. Furthermore, since we have introduced the Dirac brackets, the second-class constraints are considered to be strongly zero and therefore the FJ constraints and the Dirac ones coincide with each other. 


\section{Conflicts of Interest}

The authors declare that there are no conflicts of interest regarding the publication of this paper.

\section{Acknowledgments}

This work was supported by CONACyT under Grant no. CB2014-01/240781. The authors would like to thank R. CartasFuentevilla for discussion on the subject and reading of the manuscript.

\section{References}

[1] A. Hanson, T. Regge, and C. Teitelboim, "Constrained Hamiltonian Systems. Accademia Nazionale dei Lincei," Constrained Hamiltonian Systems. Accademia Nazionale dei Lincei, 1978.

[2] D. M. Gitman and I. V. Tyutin, Quantization of Fields with Constraints, Springer Series in Nuclear and Particle Physics, Springer, Berlin, Germany, 1990.

[3] S. Holst, "Barbero's Hamiltonian derived from a generalized HILbert-Palatini action," Physical Review D: Particles, Fields, Gravitation and Cosmology, vol. 53, no. 10, pp. 5966-5969, 1996.

[4] C. Rovelli, Quantum Gravity, Cambridge Monographs on Mathematical Physics, Cambridge University Press, Cambridge, UK, 2004.

[5] T. Thiemann, Modern Canonical Quantum General Relativity, Cambridge University Press, Cambridge, UK, 2008.

[6] J. F. Barbero G., "Real Ashtekar variables for Lorentzian signature space-times," Physical Review D: Particles, Fields, Gravitation and Cosmology, vol. 51, no. 10, pp. 5507-5510, 1995.

[7] A. Ashtekar, Lectures on Non-Perturbative Canonical Gravity, World Scientific, Singapore, 1991.

[8] R. Gambini and J. Pullin, A First Course in Loop Quantum Gravity, Oxford University Press, 2011.

[9] C. Rovelli and F. Vidotto, Covariant Loop Quantum Gravity, Cambridge University Press, Cambridge, UK, 2014.

[10] V. Bonzom and E. R. Livine, "A Immirzi-like parameter for 3D quantum gravity," Classical and Quantum Gravity, vol. 25, no. 19, 195024, 19 pages, 2008

[11] L. Faddeev and R. Jackiw, "Hamiltonian reduction of unconstrained and constrained systems," Physical Review Letters, vol. 60, no. 17, pp. 1692-1694, 1988.

[12] J. Barcelos-Neto and C. Wotzasek, "Symplectic quantization of constrained systems," Modern Physics Letters A, vol. 7, no. 19, pp. 1737-1747, 1992.

[13] J. Barcelos-Neto and C. Wotzasek, "Faddeev-Jackiw quantization and constraints," International Journal of Modern Physics A, vol. 7, no. 20, pp. 4981-5003, 1992.

[14] H. Montani and C. Wotzasek, "Faddeev-Jackiw quantization of nonabelian systems," Modern Physics Letters A, vol. 8, no. 35, pp. 3387-3396, 1993.

[15] H. Montani and J. Internat, "Symplectic analysis of constrained systems," International Journal of Modern Physics A, vol. 8, no. 4319, 1994.

[16] E. M. C. Abreu, A. C. R. Mendes, C. Neves, W. Oliveira, F. I. Takakura, and L. M. V. Xavier, "The dual embedding method in D = 3," Modern Physics Letters A, vol. 23, no. 11, pp. 829-838, 2008.
[17] E. M. C. Abreu, A. C. R. Mendes, C. Neves, W. Oliveira, and F. I. Takakura, "Duality through the symplectic embedding formalism," International Journal of Modern Physics A, vol. 22, no. 21, pp. 3605-3620, 2007.

[18] E. M. C. Abreu, C. Neves, and W. Oliveira, Internat. J. Modern Phys. A, vol. 21, p. 5329, 2008.

[19] C. Neves, W. Oliveira, D. C. Rodrigues, and C. Wotzasek, "Embedding commutative and noncommutative theories in the symplectic framework," Physical Review D: Particles, Fields, Gravitation and Cosmology, vol. 69, no. 4, 045016, 13 pages, 2004.

[20] C. Neves, W. Oliveira, D. C. Rodrigues, and C. Wotzasek, "Hamiltonian symplectic embedding of the massive noncommutative U(1) theory," Journal of Physics A: Mathematical and General, 2004.

[21] C. Neves and C. Wotzasek, "Hidden symmetries of constrained systems," International Journal of Modern Physics A, vol. 17, no. 28, pp. 4025-4042, 2002.

[22] C. Neves and W. Oliveira, "Clebsch parameterization from the symplectic point of view," Physics Letters A, vol. 321, no. 5-6, pp. 267-272, 2004.

[23] J. A. Garcia and J. M. Pons, "Equivalence of Faddeev-Jackiw and Dirac approaches for gauge theories," International Journal of Modern Physics A, vol. 12, no. 2, pp. 451-464, 1997.

[24] E. M. Abreu, A. C. Mendes, C. Neves, W. Oliveira, R. C. Silva, and C. Wotzasek, "Obtaining non-abelian field theories via the Faddeev-Jackiw symplectic formalism," Physics Letters A, vol. 374, no. 35, pp. 3603-3607, 2010.

[25] A. Escalante and M. Zarate, "Dirac and Faddeev-Jackiw quantization of a five-dimensional Stüeckelberg theory with a compact dimension," Annals of Physics, vol. 353, pp. 163-178, 2015.

[26] A. Escalante and O. R. Tzompantzi, "Hamiltonian dynamics and gauge symmetry for three-dimensional Palatini theory with cosmological constant," Journal of High Energy Physics, vol. 2014, no. 5, 2014.

[27] M. Montesinos, "Alternative symplectic structures for $\mathrm{SO}(3,1)$ and $\mathrm{SO}(4)$ four-dimensional BF theories," Classical and Quantum Gravity, vol. 23, no. 7, pp. 2267-2278, 2006.

[28] A. Escalante, "The Chern-Simons state for topological invariants," Physics Letters. B. Particle Physics, Nuclear Physics and Cosmology, vol. 676, no. 1-3, pp. 105-111, 2009.

[29] A. Escalante and C. Medel-Portugal, "Faddeev-Jackiw quantization of topological invariants: Euler and Pontryagin classes," High Energy Physics - Theory, vol. 391, pp. 27-46, 2017.

[30] D. J. Toms, "Faddeev-Jackiw quantization and the path integral," Physical Review D: Particles, Fields, Gravitation and Cosmology, vol. 92, no. 10, 105026, 9 pages, 2015.

[31] G. A. Palma, "Physical effects of the Immirzi parameter in loop quantum gravity," Physical Review D, vol. 73, Article ID 044013, 2006 


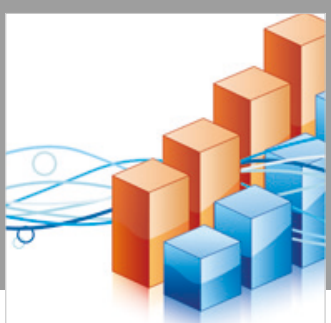

Advances in

Operations Research

\section{-n-m}
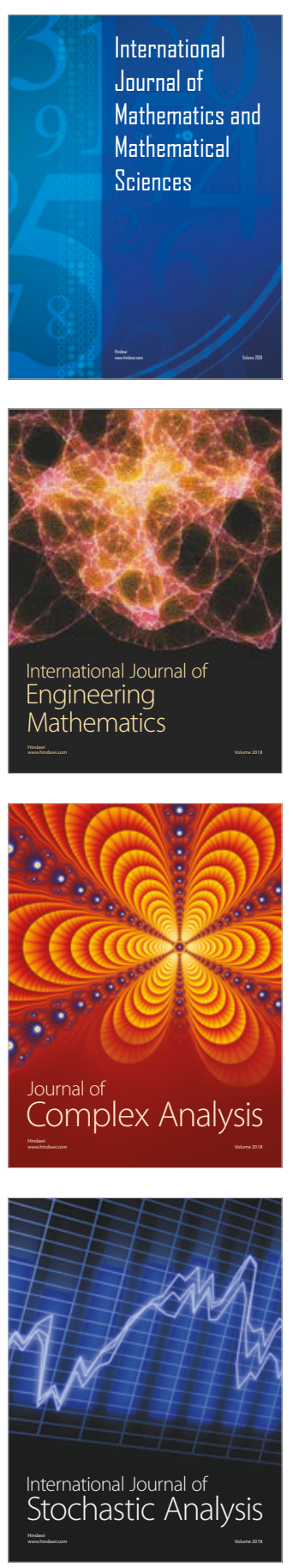
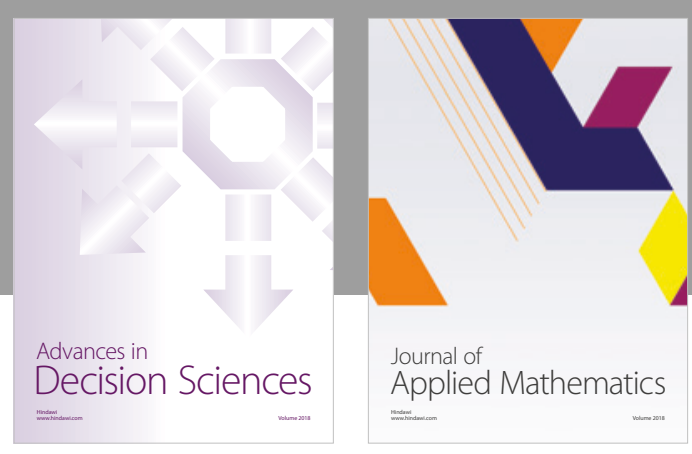

Journal of

Applied Mathematics
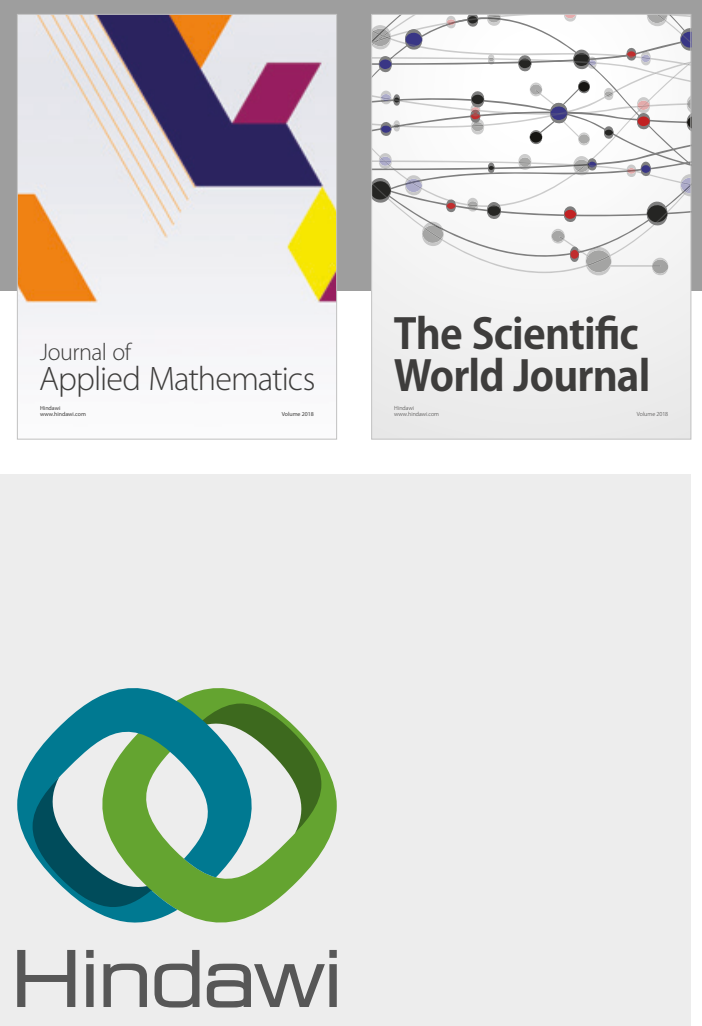

Submit your manuscripts at

www.hindawi.com

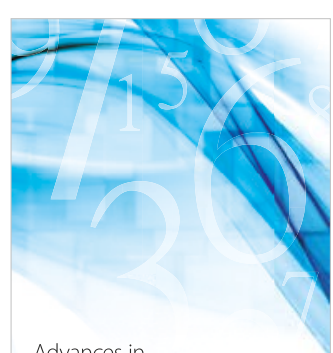

Advances in
Numerical Analysis
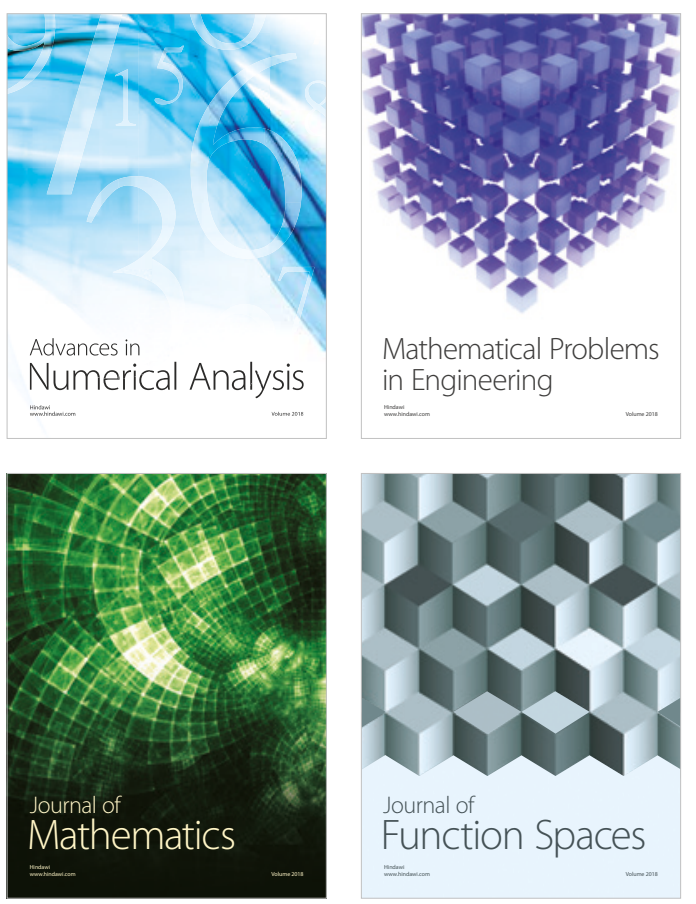

Mathematical Problems in Engineering

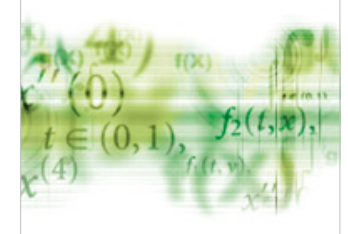

International Journal of

Differential Equations

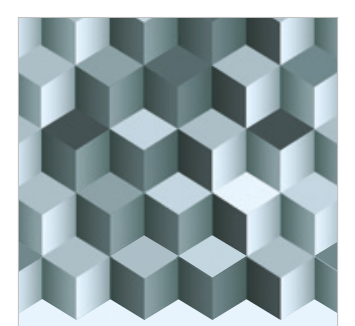

Journal of

Function Spaces

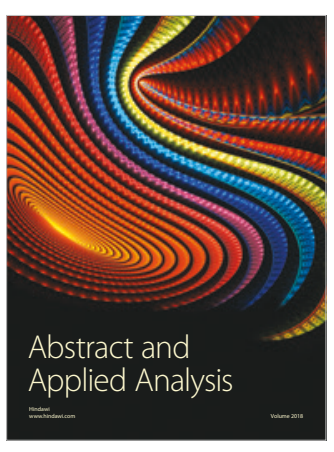

The Scientific

World Journal

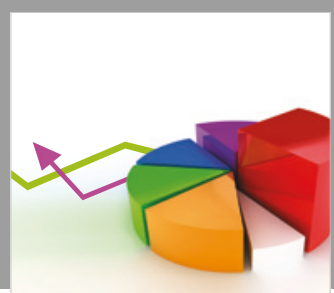

Journal of

Probability and Statistics
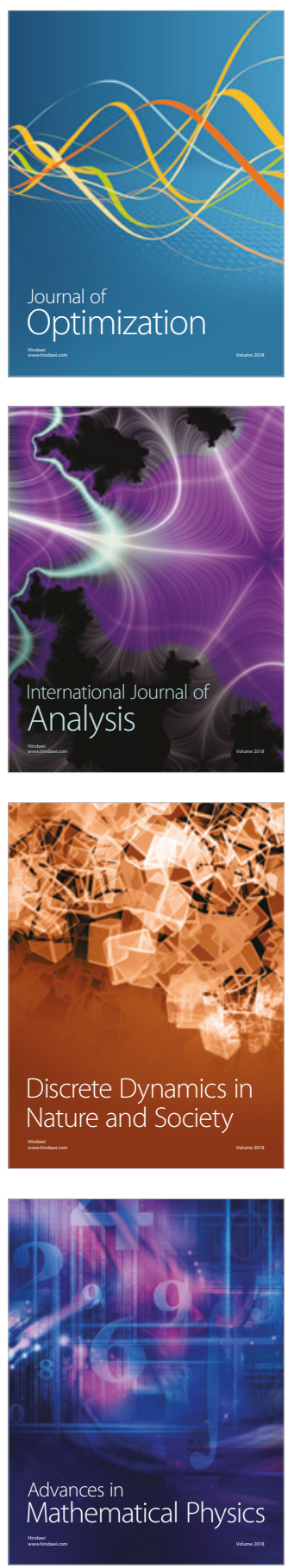